\title{
Is Continuous Chest compression CPR by Untrained Bystanders More Effective than Conventional CPR with Rescue Breathing?
}

Jorien Laermans, Anne-Catherine Vanhove, Vere Borra, and Emmy De Buck ${ }^{1}$,

${ }^{1}$ Cochrane First Aid

\section{CPR for non-asphyxial out-of-hospital cardiac arrest by untrained bystanders: to breathe or not to breathe?}

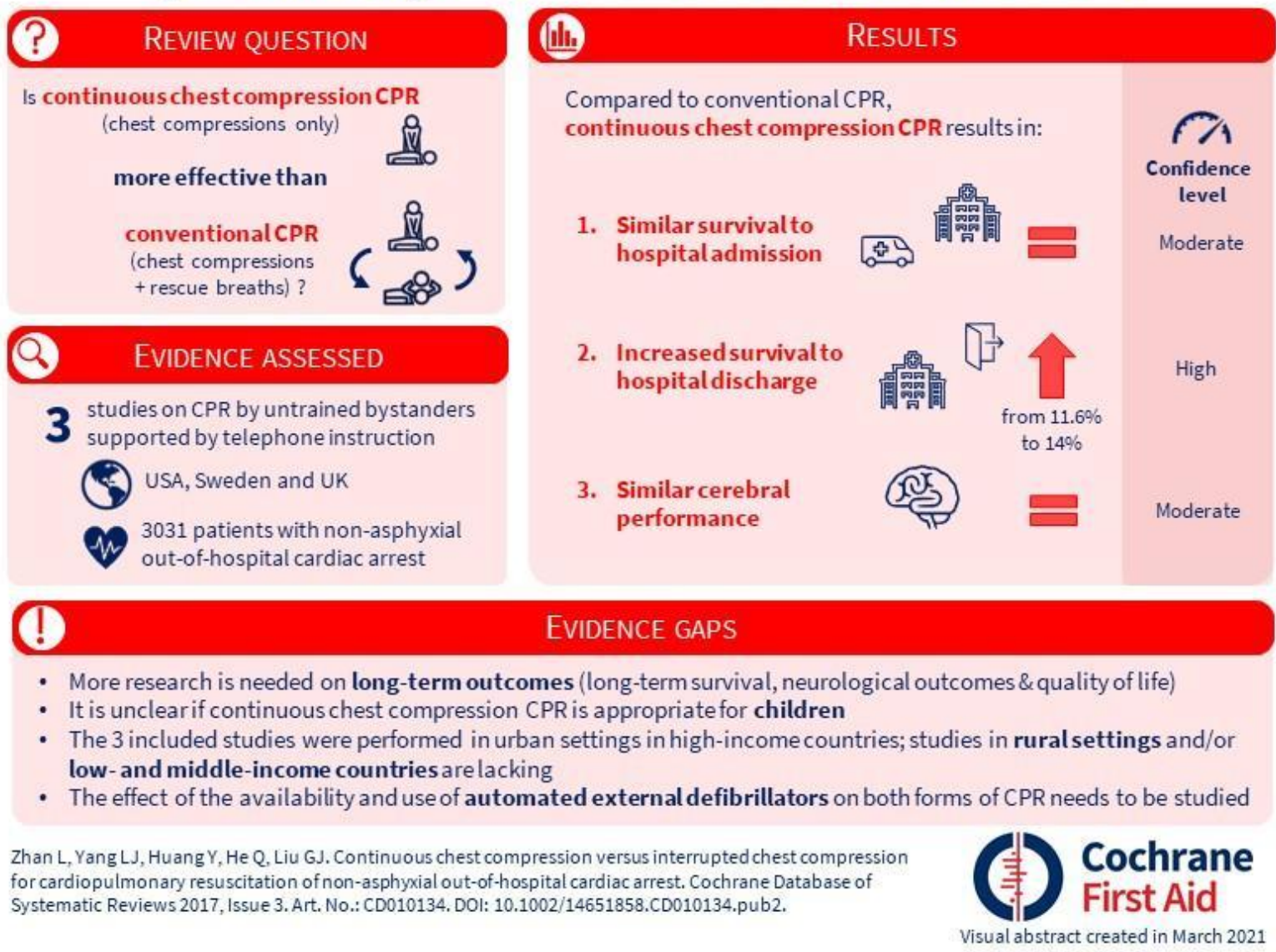

This is the first Cochrane Corner in the International Journal of First Aid Education, provided to you by Cochrane First Aid. Like any Cochrane Corner, it summarizes the findings of a Cochrane systematic review. It is meant to 
give first aid trainers, laypeople providing first aid and guideline developers direct access to highly relevant first aid-related evidence tailored to them, which they might otherwise not have access to. Additionally, this Cochrane Corner is accompanied by a visual abstract that highlights the key findings.

Cochrane First Aid aims to support Cochrane's work by disseminating Cochrane evidence to a wider audience. To find out more, we refer you to "Cochrane First Aid: the next step towards evidence-based first aid" and the Cochrane First Aid website (irstaid.cochrane.org).

This Cochrane Corner is based on a review that compares the effectiveness of continuous versus interrupted chest compression cardiopulmonary resuscitation (CPR) in case of non-asphyxial out-of-hospital cardiac arrest. The review was developed by the Cochrane Emergency and Critical Care Group and is published in the Cochrane Database of Systematic Reviews 2017, Issue 3, DOI:10.1002/14651858.CD0010134 (see www.cochranelibrary.com for information). As Cochrane Reviews are regularly updated as new evidence emerges and in response to feedback, the Cochrane Database of Systematic Reviews should be consulted for the most recent version of the review.

This Cochrane Corner and the accompanying visual abstract focus on the review's findings on CPR that is delivered by untrained bystanders supported by telephone instruction, as this is most relevant to first aid. The Cochrane review also collected the scientific evidence on CPR delivered by emergency medical services personnel. For more information on these findings, please consult the Cochrane review itself.

\section{Background}

Sudden cardiac arrest is a major global cause of death. Whereas asphyxial arrest is caused by lack of oxygen (e.g. drowning, choking), non-asphyxial arrest is usually caused by a loss of functioning cardiac electrical activity.

For victims of out-of-hospital cardiac arrest (OHCA), early CPR by bystanders is crucial for improving chances of survival to the arrival of emergency medical service personnel. There are two types of CPR:

1. Conventional CPR: this type combines chest compressions and rescue breathing (in a 15:2 or 30:2 ratio). Compressions are paused at fixed intervals to deliver rescue breaths, often by mouth-to-mouth breathing. Therefore, it is also called interrupted chest compression CPR.

2. Continuous chest compression CPR: in this type, chest compressions are not interrupted to provide ventilations, as compressions are seen as being most important for cardiac arrest victims.

While early initiation of CPR is key, (untrained) bystanders might be more reluctant to initiate conventional CPR as this involves mouth-to-mouth rescue breathing. It might also be easier to teach an untrained bystander to just perform chest compressions.

\section{Research question}

Is continuous chest compression CPR (chest compressions only, no rescue breathing) more effective than conventional CPR (chest compressions are interrupted to deliver rescue breaths) in case of non-asphyxial OHCA?

\section{Literature search}

The review authors looked for randomised controlled trials (RCTs) as well as quasi-randomised and cluster-randomised controlled trials that compared continuous chest compression CPR with conventional CPR as the control group. Studies should contain adults and/or children suffering from OHCA. Simulation and mannequin studies were not included. Outcomes of interest were survival to hospital discharge, survival to hospital admission, survival at one-year, neurological outcomes at hospital discharge and at one year, return of spontaneous circulation, quality of life, and anyadverse effects of the CPR. 
The authors searched for studies published up to February 2017 in the Cochrane Central Register of Controlled Trials, MEDLINE, Embase and Web of science. They also searched for ongoing studies in controlledtrials.com and ClinicalTrials.gov and searched conference proceedings of important meetings and abstracts. Moreover, the reference lists of the International Liaison Committee on Resuscitation evidence worksheets were searched, and experts in the field were contacted.

\section{Results}

The authors identified three relevant RCTs, performed in urban areas of the USA, Sweden and the UK. One study only looked at adult OHCA patients, whereas the patients in the second study had to be at least 8 years old. The third study did not describe the minimum age of the patients. The mean age of the patientsvaried between 63 and 68 years old.

In all studies, after the dispatcher had determined that the patient showed signs of reversible OHCA and that the untrained bystanders were willing to be instructed, the dispatcher provided instructions by phone on either continuous chest compression or conventional CPR.

These studies indicate that, compared to conventional CPR, continuous chest compression CPR:

- increases survival until discharge from 11.6 to $14 \%$ (3 studies, 3031 patients, high-certainty evidence);

- probably results in little to no difference in survival to hospital admission (1 study, 520 patients, moderate-certainty evidence) and in neurological outcomes at hospital discharge (1 study, 1286 patients, moderate-certainty evidence).

There were no data available on survival at one year, return of spontaneous circulation, quality of life,or adverse effects of CPR.

\section{Conclusion}

After non-asphyxial OHCA, bystander-administered continuous chest compression CPR supported by telephone instruction increases the proportion of people who survive to hospital discharge compared with conventional CPR. No significant differences were found for survival to hospital admission and neurological outcomes at discharge, but these outcomes were reported in only one study each and the number of patients were low.

\section{Implications for first aid practice and research}

This review indicates that in case of non-asphyxial OHCA where CPR is delivered by an untrained bystander under telephone instruction from the emergency services, continuous chest compression CPR leads to 25 more people per 1000 surviving to hospital discharge, compared to conventional CPR. However, more research is needed to assess the effects of the different treatments on long-term outcomes, including long-term survival, neurological outcomes and quality of life. It also remains unclear whether continuous chest compression CPR is appropriate for cardiac arrest in children. Moreover, the three studies included in this systematic review were all performed in urban settings in high-income countries, which limits the generalizability of the results. Studies certainly need to be performed in rural settings, as well as in low- and middle-income countries to know whether continuous chest compression or conventional CPR are better in these conditions. Finally, the influence of the availability of automated external defibrillators (AEDs) and actual AED use on the effects of both continuous chest compression and conventional CPR needs to be investigated. 


\section{Acknowledgements \& Funding}

Jorien Laermans, Anne-Catherine Vanhove and Vere Borra are Field Coordinators of Cochrane First Aid. Emmy De Buck is the director of the Field.

\section{References}

Zhan L, Yang LJ, Huang Y, He Q, Liu GJ. (2017). Continuous Chest Compression Versus Interrupted Chest Compression for Cardiopulmonary Resuscitation of Non-asphyxial Out-of-Hospital Cardiac Arrest. Cochrane Database of Systematic Reviews (3). Art. No.: CD010134. DOI:

10.1002/14651858.CD010134.pub2 